\title{
Using gamma spectrometry indicators to detect and quantify fission products changes in irradiated fuel
}

\author{
L. Loubet, Th Martella, CEA/DEN/DEC/SA3C, Cadarache Centre, Fuel Study Department, Bt 315, 13108 \\ St Paul Les Durance, France Tel:+33 442254 572, Fax: +33 442257 042, \\ E-mail:laurent.loubet@cea.fr
}

\begin{abstract}
A new analysis method based on gamma scanning of fission products on irradiated rods is presented. Indicators calculated from this method can be used for the qualitative treatment and comparison of irradiated rods from PWR, SFR or and MTR. Differences in the behavior of fission products (FP) can thus be quantified. Phenomena such as migration or geometrical changes in pellets should thus benefit from these accurate, yet quickly and easily achievable results.
\end{abstract}

\section{CONTEXT OF GAMMA SPECTROMETRY MEASUREMENTS ON IRRADIATED FUEL}

Non-destructive examinations (NDE) performed in the LECA STAR hot cells provide key data on irradiated fuel rods and pins. These non-destructive examinations are performed on benches such as Megafox for long rods up to $4.5 \mathrm{~m}$, or Vendaum for pins or experimental rodlets up to $2.7 \mathrm{~m}$. The irradiated rods or pins are moved on these benches in an accurate axial and angular direction in front of motionless sensors. Among the non-destructive examinations available, gamma spectrometry is used to collect information on the fuel inside the cladding by means of gamma rays emitted from the fission products in the pellets. In this case, the sensor is a high-purity gamma detector located outside the hot cell. A specific collimator inserted through the wall of the hot cell allows to scan an axial slice of the rod (Figure 1).

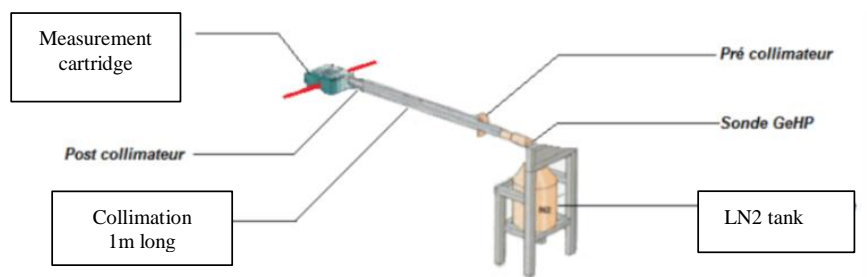

Fig. 1. Gamma spectrometry on the Megafox bench

The bench's instrumentation \& control (IC) system ensured the accurate step-by-step displacement of the irradiated rod inside the hot cell. Generally speaking, one measurement point per mm, is selected, e.g.. nearly 4,500 spectra for a PWR rod. A region-of-interest (ROF) treatment is used for each spectrum to provide the axial scan of fission products such as ${ }^{137} \mathrm{Cs},{ }^{154} \mathrm{Eu}{ }^{106} \mathrm{Rh},{ }^{95} \mathrm{Nb}$ along the rod. The position and the length of the fuel column and the pellets can be determined on the basis of these gamma scans. An example of these gamma scans can be seen in Figure 2 below.

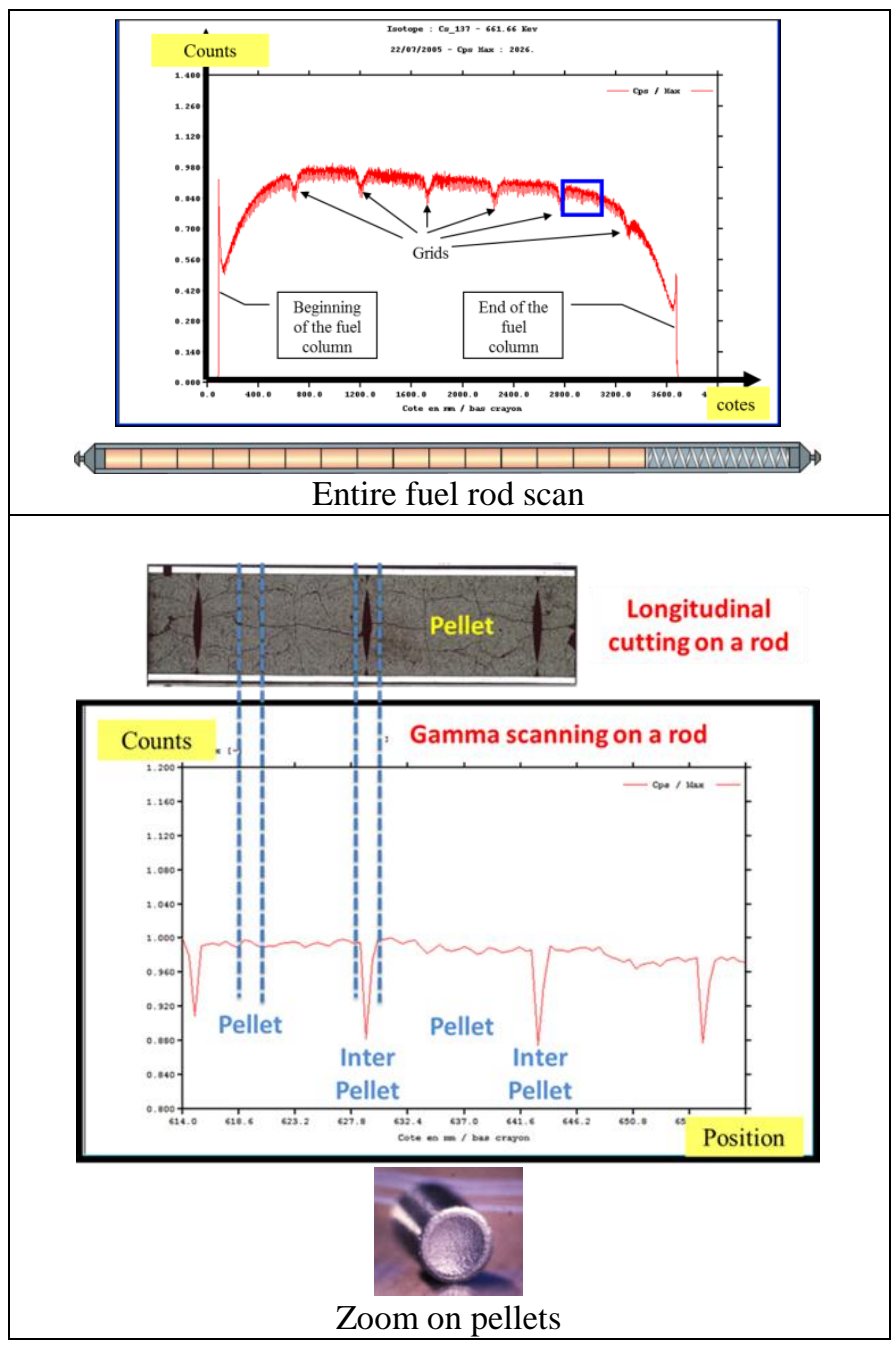

Fig. 2. Gamma scans on an irradiated long rod and a zoom on the pellets

The laboratory database already contains gamma scans on hundreds of rods and pins with different types of fuels, cladding and irradiation conditions (nominal and non-nominal conditions). 


\section{DESCRIPTION OF THE CALCULATION METHOD AND INDICATORS (PATENTS)}

The initial data are available in the form of files with a counting rate measured at each axial position on the rod (typically 1 point per $\mathrm{mm}$ ) for each fission product selected. In the end, this produces scan such as the one represented above.

As can be seen in the zoom of such a scan, the gamma scan of fission products such as ${ }^{137} \mathrm{Cs}{ }^{154} \mathrm{Eu},{ }^{106} \mathrm{Rh},{ }^{95} \mathrm{Nb}$, etc. reveals a specific shape due to the shape of the pellets themselves and to the measuring conditions through a collimator. Between two adjacent pellets, a decrease in the counting rate of all the fission products can be seen due to the lack of matter in the dishing and chamfer area of the interpellet.

An in-house computer program had been developed to collect more information from the scans. The elementary representative unit chosen is equivalent to one pellet with its chamfers and dishing. In fact, two adjacent half pellets and their chamfer and dishing as can be seen in the picture below.

This program computes a first step from the data in order to determine the position of the pellets along the rod. To do this, it starts with the distribution of a statistically representative FP. Thanks to the choice of an appropriate discriminator, it is able to categorize points corresponding to the pellet $(\mathrm{P})$ with a counting rate $\mathrm{Y}$, from points corresponding to the inter-pellet (IP) with a counting rate of Y-dy.

After this pre-processing, each measurement point along the fuel column is allocated a Boolean attribute: pellet/inter-pellet.

The measured counting rate, (IP_n)measurement, for each inter-pellet $\mathrm{n}$ of the fuel column is then obtained. The measured counting rates, $\left(\mathrm{P} \_n\right)$ measurement and $\left(\mathrm{P} \_n+1\right)$ measurement, for the two adjacent half pellets on both sides of the $n$ inter-pellets are also obtained (Figure 3 ).

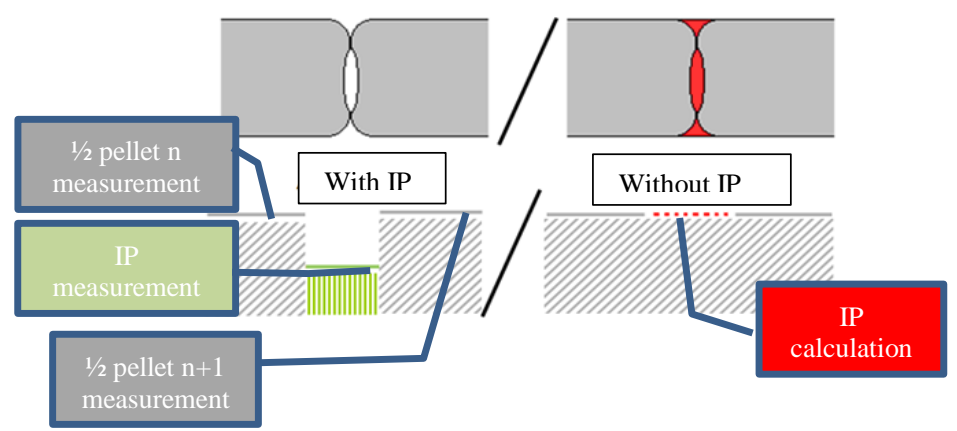

Fig.3. Principle of calculation at the inter-pellet

Another calculation for this indicator is to determine the counting rate for each inter pellet, $n$, that would have been obtained at this position without chamfer and dishing: (IP) calculation.

After this step, it was possible to finally compare (IP_n) measurements and (IP_n) calculations for each fission product and for each inter-pellet of the rod. For each inter-pellet, a local indicator $\left(\mathrm{R} \_\mathrm{n}\right) \mathrm{FP} \mathrm{i}$ is calculated as the relative difference between (IP_n)measurement and (IP_n)calculation for each fission product selected.
An indicator of 1 corresponds to a configuration without a decrease in the counting rate in the inter-pellet zone. Naturally, due to chamfer and dishing, (R_n)FP_i is $<1$.

In the first order, uncertainties depend on the statistical precision of the fission product counting.

Individual values of $\mathrm{R}$ for each IP of a rod and a mean value for the entire rod are obtained.

\section{FIRST RESULTS WITH THE INDICATOR}

Based on this principle, the mean indicators for one real entire rod (nearly 300 pellets) and for 3 fission products: (R)137Cs, (R)106 Rh and (R)95Nb have been calculated.

- $\quad(\mathrm{R}) 137 \mathrm{Cs}=0.958+/-0.011$

- $\quad(\mathrm{R}) 106 \mathrm{Rh}=0.959+/-0.015$

- $\quad(\mathrm{R}) 95 \mathrm{Nb}=0.954+/-0.018$

These results show that such an indicator provides a relevant and coherent quantification of the usually observed decreasing counting rate in the inter-pellet zone.

As seen before, this decrease in the fission product counting rate in the inter-pellet zone is due to the geometrical conditions (chamfer, dishing, etc.). The theoretical value of $\mathrm{R}$ in those measurement conditions (chamfer, dishing, collimation, etc.) can be estimated. A value of $R=0.96$ was obtained. This result confirms and qualifies the calculation.

\section{CAPACITIES OF THE INDICATORS}

In a more global approach, the indicators were used more generally to compare four similar rods used for this test case. The results can be seen in Figure 4 below.

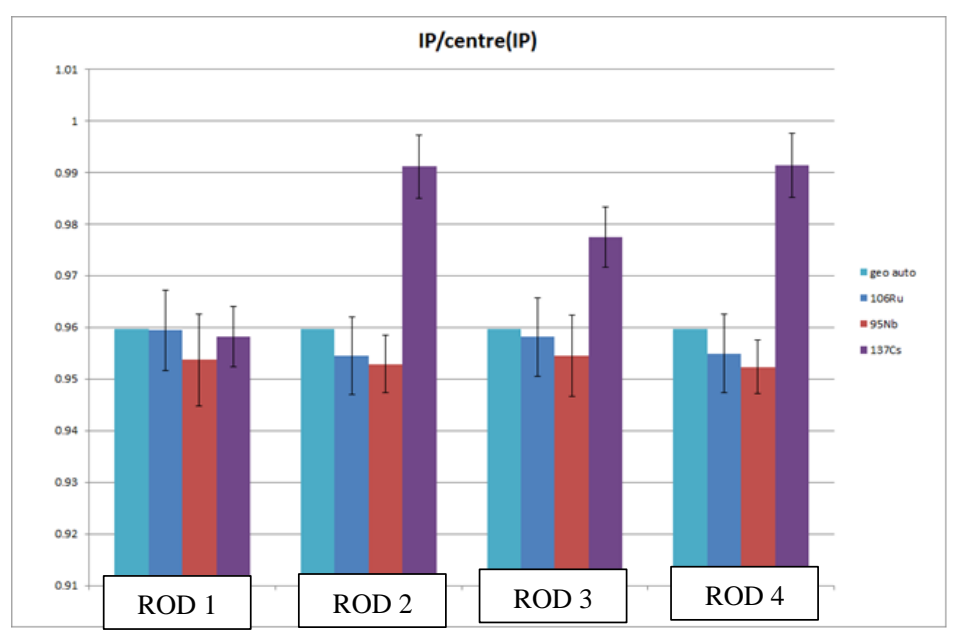

Fig.4. Comparing the results of mean indicators for several FPs of a long rod

This mean use of the indicators made it possible to compare and detect global differences between fission product behaviors in irradiated rods.

After these mean calculations, a focus can be done on local results. The nominal area is the range where one should statistically find $95 \%$ of the $\mathrm{R}$ values for the selected fission product. This can be seen for the rod used in case 1 for the $95 \mathrm{Nb}$ indicator in Figure 5 below. 


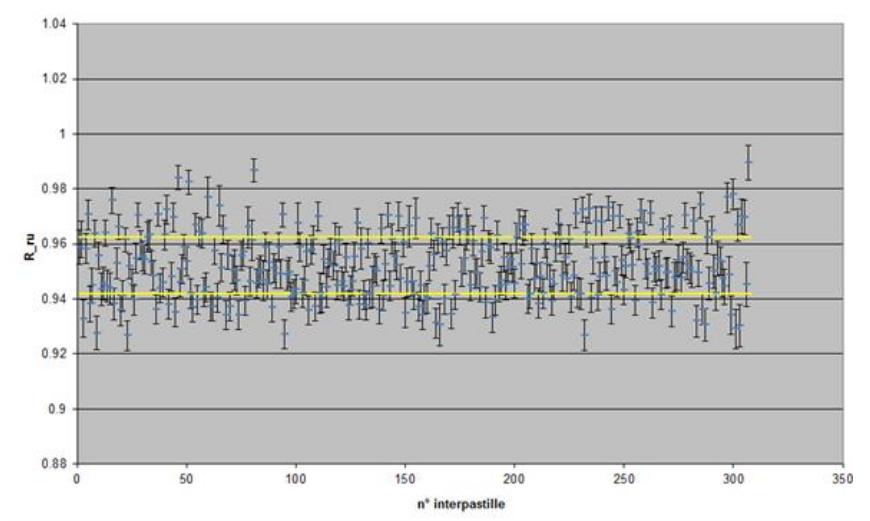

Fig.5. Results of local (R)95Nb in the pellets of a long rod

The calculations of these indicators were performed for two different rods, case 1 and case 2 (Figure 6).

In these specific cases, such as case 2 below, local differences can clearly be seen between the results of R for 137Cs.

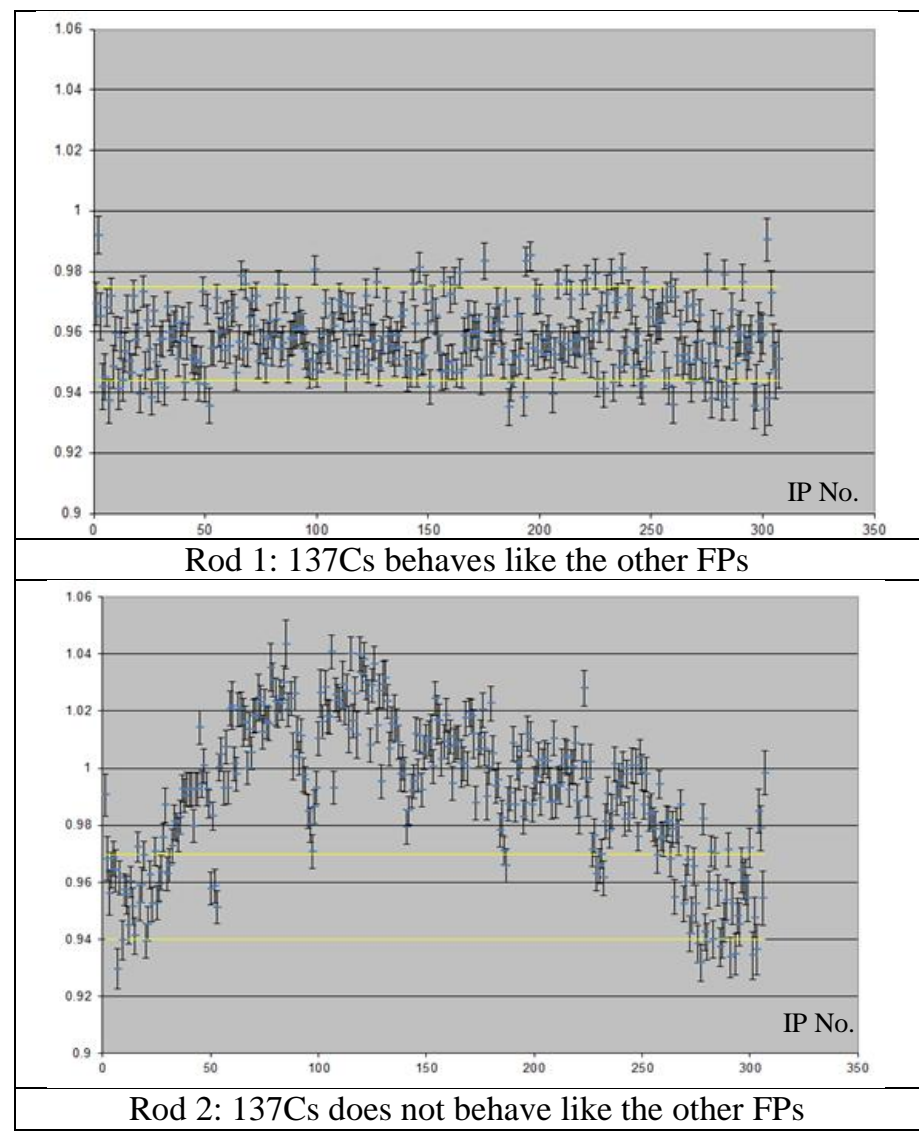

Fig.6. Comparing the results of local (R)137Cs in the pellets of 2 long rods

This is in total agreement with the overall observations. Such calculations now allow to locate, quantify and compare fission product behavior all along a rod, a rodlet or a pin.

These results can thus be used to quantify qualitative observations of rods when the decreasing counting rate at the inter-pellet due to the pellet geometry and measurement shortcomings is no longer observable on ${ }^{137} \mathrm{Cs}$ scans and can even become an increasing counting rate. In some cases, this can be explained by the migration of cesium, which moves to the cold sides of the pellet. This phenomenon is mainly associated with an increase in the pellet's temperature.

As illustrated by the blue curve (case No. 2) in the graph below, a different shape can be observed in ${ }^{137} \mathrm{Cs}$ scans in such specific cases. In these cases, the ${ }^{137} \mathrm{Cs}$ scan can be locally different from the usual fission product distributions of Fission Products but wasn't quantified yet.

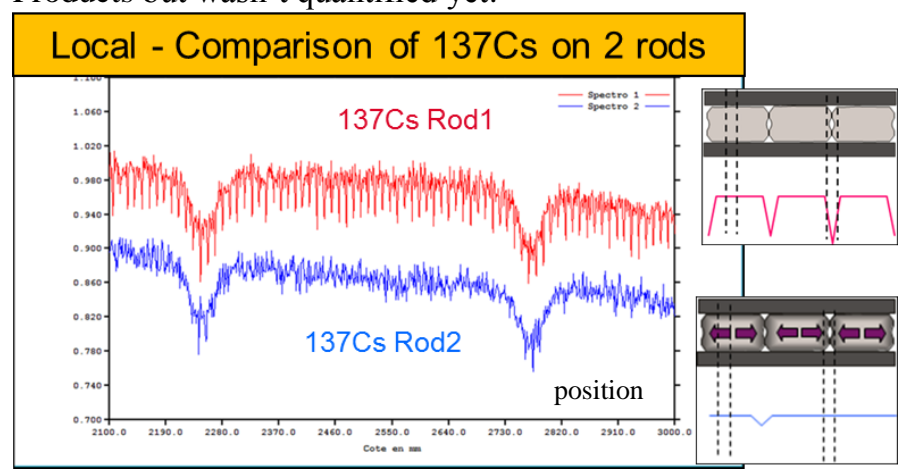

Fig.7. Differences between 137Cs scans

\section{CONCLUSIONS AND FUTURE PROSPECTS}

Based on the decreasing counting rate in front of inter-pellets, indicators are determined from gamma results on fission products from irradiated rods. Those indicators have been qualified and compared with the theoretical value due to the geometrical conditions of this measurement (chamfer, dishing, and collimation). Used in a local way, these indicators make it possible to detect and quantify differences in the ${ }^{137} \mathrm{Cs}$ behavior, e.g. all along a rod, a rodlet or a pin, under nominal or non-nominal conditions. More generally, these indicators can also be used to compare rods between themselves.

Optimization of the indicators is in process in order to increase their sensitivity.

This being said, these results are already quickly and easily achievable from gamma measurements, which can then be easily correlated to other observations obtained from destructive examinations, puncturing and calculations that have either already been performed or are pending.

Since fission product migration such as cesium is enhanced by a temperature increase in the pellets, the indicators should be directly associated with the local temperature in some cases.

In the future, thermally activated phenomena such as geometrical changes in the pellets and fission gas releases should also be indirectly inferred from those indicators.

\section{ACKNOWLEDGMENT}

We would like to thank J. NOIROT for all the help and support he provides on a daily basis.

\section{REFERENCES}

[1] Patent No. 14179615.1 -1556. 26/09/2014.

[2] Patent No. 14179628.4 -1556. 20/09/2014 